\title{
ARTIFICIAL INTELLIGENCE TO SUPPORT BULGARIAN CROP PRODUCTION
}

\author{
Lyubka Atanasova Doukovska \\ Institute of Information and Communication Technologies, \\ Bulgarian Academy of Sciences, \\ Acad. Georgy Bonchev Str., Block 2, 1113 Sofia, Bulgaria, \\ e-mail: lyubka.doukovska@iict.bas.bg
}

\begin{abstract}
Targeted scientific and applied research on the application of artificial intelligence and digital technologies in agriculture reduces costs for farmers, improves soil management and water quality, reduces the use of fertilizers and pesticides, and reduces greenhouse gas emissions, improving biodiversity and creating a healthier environment for farmers. This paper presents the results of research aimed at the application of artificial intelligence to support Bulgarian crop production. Results obtained within the framework of the project BG PLANTNET "Establishment of National Information Network GenBank - Plant genetic resources" and the National Research Program "Smart crop production" are presented. A concept for building an intelligent platform for agricultural process management is presented.

Keywords: artificial intelligence, intelligent agriculture, intelligent systems, virtual physical space, cyber-physical-social system, Internet of Things, ambient intelligence, intelligent agents.
\end{abstract}

\section{INTRODUCTION}

Artificial intelligence is one of the main drivers of digital transformation in Europe and an important factor in ensuring the competitiveness of the European economy and high quality of life. Artificial intelligence will significantly contribute to the development of modern, efficient, knowledge-based agriculture, which will increase the quality of food and conserve natural resources.

The National Scientific Program "Intelligent Crop Production" was approved by a decision of the Bulgarian Council of Ministers No 866 of November 26, 2020 [1]. The topics and goals of the program according to the National Strategy for Development of Scientific Research until 2030 refer to one of the priorities for targeted basic research and the following priority areas for development of applied research:

DOI: 10.7546/EngSci.LVIII.21.04.03 
- Improving the quality of life - food, health, biodiversity, environmental protection, urban environment and transport, etc.;

- Mechatronics and clean technologies;

- Information and communication technologies;

- Health and quality of life. Prevention, early diagnosis and therapy, green, blue and eco-technologies, biotechnologies, eco-foods.

The obtained results are planned to support the future implementation of the Strategic Plan in the new Common Agricultural Policy 2021-2027, based on the analysis of the needs and requirements of our country in the field of agriculture, as well as the adopted European strategy "From farm to table" accelerating the transition to a sustainable food system.

The Agricultural University-Plovdiv is the coordinator of the National Research Program that is structured in four Components:

- Component 1. Digital, IoT and robotic technologies in crop production. Construction of infrastructure for intelligent crop production;

- Component 2. Diagnosis and prognosis through artificial intelligence;

- Component 3. Intelligent management system for agricultural processes;

- Component 4. Artificial intelligence and digital technologies - Drive for innovative management systems, sectoral dynamics and change in quality of life.

Each component consists of several work packages, the teams of which include scientists from nine scientific organizations - partners in the program.

Participants in the team from the Institute of Information and Communication Technologies at the Bulgarian Academy of Sciences are included in the execution of the tasks in two of the Components of the program (Components 1 and 3), which are presented in the following work packages:

- WP 1.3. Intelligent Agriculture Infrastructure;

- WP 3.2. Convergence of the Internet of Things and Big Data in Intelligent Agricultural Process Management;

-WP 3.3. Virtual Operational Center for Intelligent Agriculture Management;

- WP 3.4. Blockchains for Intelligent Agriculture.

Each work package consists of several tasks. The scientific research in this program will support the development of agriculture as a high-tech, sustainable, highly productive and attractive area of the economy, which will contribute to improving the living conditions of farmers and rural areas in general.

In the last year, various projects were launched, the aim of which was to develop platforms for intelligent agriculture. The FaST digital services platform (https://fastplatform.eu/) aims to provide EU farmers with possibilities for 
agriculture, sustainability, and the environment. The platform is supported by the European Commission's DG Agriculture and Rural Development, by the EU Space Programme (DG DEFIS), and by the EU ISA2 Programme (DG DIGIT).

This paper presents the idea of creating an original platform for intelligent Bulgarian crop production adapted for the needs of Bulgarian farmers - Smart Agriculture Platform.

\section{RELATED WORKS}

One of the major challenges that smart agriculture is expected to address is the efficient use of water resources. The conservation and the efficient use of clean water is a long-term strategy worldwide. Modelling smart agriculture systems is an important factor because the processes there are very slow, and sometimes it takes a year or more for a full crop cycle. At the same time, a large amount of data is usually needed to make informed decisions. This determines the importance of developing appropriate systems through which to simulate, generate, optimize and analyze various possible scenarios and prepare appropriate plans. An infrastructure known as Virtual-Physical Space (ViPS) adapted for agriculture is presented in [2]. The space supports integration of the virtual and physical worlds where analysis and decision making are done in the virtual environment and the state of the physical objects (things) of interest is also taken into account at the same time. Special attention is paid to the possibilities of modelling an irrigation system. An ambient-oriented approach has been adopted, using the Calculus of Context-aware Ambients formalism as the basic tool for modelling agriculture processes. Furthermore, the supporting platform is briefly presented. Active components of the platform are implemented as intelligent agents known as assistants. Users (agriculture operators) are serviced by personal assistants. Currently, the presented modelling system is deployed over a two layered system infrastructure in the region of Plovdiv city. Plovdiv is the center of vegetable production in Bulgaria.

Paper [3] represents the view of the authors about the needs and the implementation of a distributed system among all institutes in Bulgaria for management of plant genetics resources according to the standards defined by the EURISCO catalogue.

An adaptation of ViPS to build an intelligent infrastructure for agriculture in the Plovdiv region termed "Agriculture 2.0 - Plovdiv" is presented in [4]. The main challenge is the virtualization of "things" from the physical world, as well as related events, time and spatial aspects. To present the spatial aspects 
of things an Ambient-Oriented Modeling approach is used. An animated simulator is created by adapting an ambient-oriented interpreter to model some scenarios implementing basic services in the space.

Paper [5] presents the development of ontology for plant genetic resources in the Genebank of the Institute of Plant Genetic Resources in the town of Sadovo. The structure of the ontology and metadata are exhibited along with each of the concepts and properties in order to present knowledge about plant genetic resources using the capabilities and benefits of ontologies. The ontology was developed on the basis of the taxonomy for plant genetic resources and the EURISCO European standard.

An infrastructure for smart agriculture is presented in [6]. The basic components of the infrastructure are also presented. The kernel component is the Operative Center consisting of personal assistants. The purpose of this center is to be the institution for operational management of the whole infrastructure. The development of a personal assistant as a belief-desire-intention intelligent agent is presented in more detail. Some implementation issues of the personal assistant are also discussed in this paper.

The model presented in [7] enables integration and interconnection between different modules of the developed "Agriculture 2.0 - Plovdiv" system. The use of blockchain technologies provides the necessary level of security, completeness and reliability in the input and use of information related to the Bulgarian Genebank. The main tasks in the environment development are the digitalization of the Bulgarian Genebank and the creation of a web portal providing information and services to different groups of users.

A multistage algorithm for automatic analysis of documents from the area of plant genetic resources is proposed in [8]. The determination of the significant words in the document and the type of its content is carried out based on the object-oriented ontologies in the field. The documents are classified into related and not related to the studied subject area - agriculture and plant genetic resources of Bulgaria, by applying software instruments for classification, testing and evaluation.

A multi-step application-oriented decision-making model for digital risk management of disruptive technologies in agriculture is presented in $[9,10]$. The model is a conceptual framework of known risk management procedures and multicriteria selection instruments. The model is tested with data on nine disruptive technologies and eight digital risk categories. The risk data is assessed with the quasi-multicriteria algorithm SIGMA. The tests demonstrate both the application of the model, the approach to the analysis of identified digital risks, and the risk profiles used in the decision-making process. 
The modern educational spaces shown in [11] integrate data from the Cyber-Physical-Social System (CPSS) space, which can be analyzed using various techniques. This enables the implementation of intelligent applications and services.

Paper [12] proposes a method of making optimal decisions with risks in the very important task of transporting agricultural products on a network of arbitrary configuration. The method takes into account both the specific network properties and the probability of adverse events and the risks to the individual sections of the network in the implementation of these services. The proposed method minimizes not only the total cost of transporting agricultural produce but also the insurance costs to cover the risks. A complete numerical modelling of decision-making and transportation of agro-products on a network of arbitrary configuration is implemented, whereby both the cost of transport and the coverage of emerging risks are minimized by the proposed method.

A model of an intelligent system for the recognition of wheat crops pests is introduced in [13]. The recognition allows the species of weeds, their density, the various diseases and vermin of beneficial plants to be defined, as well as the exact location of objects in the field. The recognition technology is based on a convolutional neural network and on the independent work of a drone.

Paper [14] outlines the prerequisites for the introduction of a model for the integration of an intended interface platform for storing information regarding results and background in a blockchain network. The results are based on the research and the development of a concept for use of the existing infrastructure of the Institute for Plant Genetic Resources "Konstantin Malkov", Sadovo, Bulgaria. The described model is based on existing concepts from the Blockchain research community and the resulting architecture can be used to build a class of applications based on this concept.

Bulgaria is one of the richest countries in plant species. Many of these species are disappearing for various reasons - climate change, artificial modification, or adaptation due to different climate conditions. One of main tasks is to preserve and protect these species over time. A large number of scientific organizations and institutes study and store plant genetic resources in gene banks under special conditions. One of them is the Institute for Plant Genetic Resources in the town of Sadovo, where the largest gene bank in Bulgaria is located. Processing information for storage and management of plant genetic resources is performed by using the system developed and designed for the needs of the institute, which is called the GenBank Management System [15]. 
The documentation of germplasm accessions stored in the Bulgarian seed Genebank is presented in [16], as well as the new approaches corresponding with the international agreements addressed the conservation, sustainable use and access and benefit-sharing of these resources. The survey is based on the national register of plant genetic resources and the European Searching Catalogue EURISCO. The National Inventory of Bulgaria includes 69,644 accessions according to EURISCO. Among those 17,978 accessions are characterized with Bulgarian origin. During the period 1982-2021 the fund of the National Genebank in Sadovo has been enriched with 53,474 seed accessions. 10,715 local accessions and wild crop relatives from home gardens and natural habitats within the country have been collected through expeditions. There are 36,712 genotypes introduced by the international free exchange. 6,047 breeding materials are registered: lines and advanced varieties. Collections of cereals, grain legumes, oil and industrial crops, forages, vegetables, medical and aromatic plants have been created. All seed accessions are listed according to the international standards of FAO/Bioversity Multi-Crop Passport Descriptors.

\section{INTELLIGENT CROP PRODUCTION}

In the last decades, with the great break-throughs in science, technology and engineering, people have been interacting more and more intensively with the surrounding physical world. The modern technologies of Cyber-Physical Systems (CPS) and the Internet of Things (IoT) contribute significantly to the evolution of computer interconnection [17-22]. They integrate the dynamics of physical processes with those of software and communication, providing abstractions and techniques for modelling, designing and analyzing complex integrated systems [23]. Cyber-physical systems closely connect and coordinate computational (cyber) and physical resources, i.e. systems that maintain close integration between computing, communication and control, interacting with the environment in which they are located [24].

Agriculture is becoming an increasingly relevant area for conducting research and practical developments in the field of Cyber-Physical-Social System, Cyber-Physical System and Internet of Things. These three relatively new and interconnected technologies provide a variety of unthinkable possibilities of building infrastructures for intelligent farming. As water supplies become scarce due to climate change, there is an urgent need to irrigate more efficiently in order to optimize water use. Effective pest control and minimizing the risk of environmental pollution by pesticides are the other major 
challenges of smart farming. In the same time, agriculture usually has a huge number of crops and machinery that grow and operate in the wide geographic areas in which they are located. They are subject to operational factors often beyond their control, such as the amount of sunlight or rainfall that they receive, or the temperature fluctuations. In this context, agriculture is ripe for the adoption of new technologies to help monitor and manage assets on a granular level, and the development of intelligent CPSS- and IoT-based agriculture infrastructures would be of great benefit [25]. In the specialized literature, a great number of examples of such solutions and systems can be found.

An agricultural cyber-physical-social system integrating three worlds that in turn are mapped to the cyber space and physical space is presented in [26]. The physical world consisting of crops, their environment and control devices, is mapped into cyberspace through various IoT techniques. The mental world includes a wide variety of data in the form of producers' experience and knowledge, customer preferences, timing, quantity and application of fertilizers. In the artificial world of the system, crop models are maintained and decisions are made that affect the physical world through the control devices. The functionality of the proposed architecture is demonstrated with a case study on the solar greenhouse. Architecture for monitoring soil humidity and temperature in small farms divided in regions is described in [27]. The proposed system monitors soil moisture, humidity and temperature in the relevant areas using wireless sensing networks and IoT methods, and if needed it sends reports to the end users. The farmers can more effectively plan crop cultivation, harvesting, irrigation and fertilization by help of reports information. An infrastructure consisting of five nodes with one or more sensors is proposed in order to assist precision agriculture [28]. The data acquisition from various sensors allows monitoring of climate elements such as soil moisture, air quality, temperature and humidity, precipitation intensity, precipitation level, wind speed and direction, light flux and atmospheric pressure. In addition, a web page has been developed where the user can view the climatic conditions of cultivation or afforestation. Another architecture and an application prototype for precision farming using a wireless sensor network with an IoT cloud is proposed in [29].

A useful review of current research and research projects in the field of big data to analyze and solve various significant issues in agriculture is presented in [30]. The review highlights the large capabilities of large data for intelligent and precision agriculture. Furthermore, it shows that the availability of hardware and software, techniques and methods of analyzing large data as well as the increasing openness of data sources will encourage further academic 
research, public sector initiatives and business initiatives in the agricultural sector.

The IoT technology is built on the concept of "smart" objects that are able to autonomously and proactively collect, store and transmit data. According to this concept, Virtual-Physical Space can receive data from the physical world, as well as share knowledge, plan and make decisions in the virtual world.

In recent years, the team in the program has been developing a reference infrastructure called ViPS [33], which can be adapted to develop CPSS applications in various application areas, Fig. 1.

An agent-oriented approach is applied to build the genetic infrastructure. The fundamental components of ViPS are implemented as rational practical reasoning agents. Personal assistants will assist users in working with domainspecific adaptations of ViPS.

Our idea is ViPS to operate as a space, i.e. the active components are autonomous and proactive and they interact through messages rather than through strictly defined interfaces. However, the individual components can be considered as located in three functional layers: ViPS User Interface, ViPS Middleware, and ViPS Physical World.

Usually, it is hard for users to work in a heterogeneous space such as a ViPS-like application due to the inherent complexity. For this reason, personal assistants operating on the behalf of the users are aware of their needs to select or prepare appropriate scenarios for the execution of users' requests. Furthermore, personal assistants can manage and control scenario execution interacting with the ViPS middleware. Besides, the personal assistants exercise control of personal access to a ViPS-like space, Fig. 1.

The work of the personal assistants is closely related to the Event Engine. The personal assistant's lifecycle is supported by a user's personal timetable. The personal timetable presents the activities that the user has to perform in a certain period of time. The entries on the timetable are presented as domain-specific events corresponding to a suitable activity. Furthermore, the events can be used for a specification of policies that in turn present space operative scenarios.

The personal assistants are implemented as BDI rational intelligent agents [34]. The BDI lifecycle incorporates two steps known as deliberation and means-ends reasoning. During the deliberation, a personal assistant generates its current goal while in the next step (means-ends reasoning) a plan for the goal achievement is prepared. Usually, the plan is launched when a corresponding event occurs, i.e. by means of the Event Engine. 


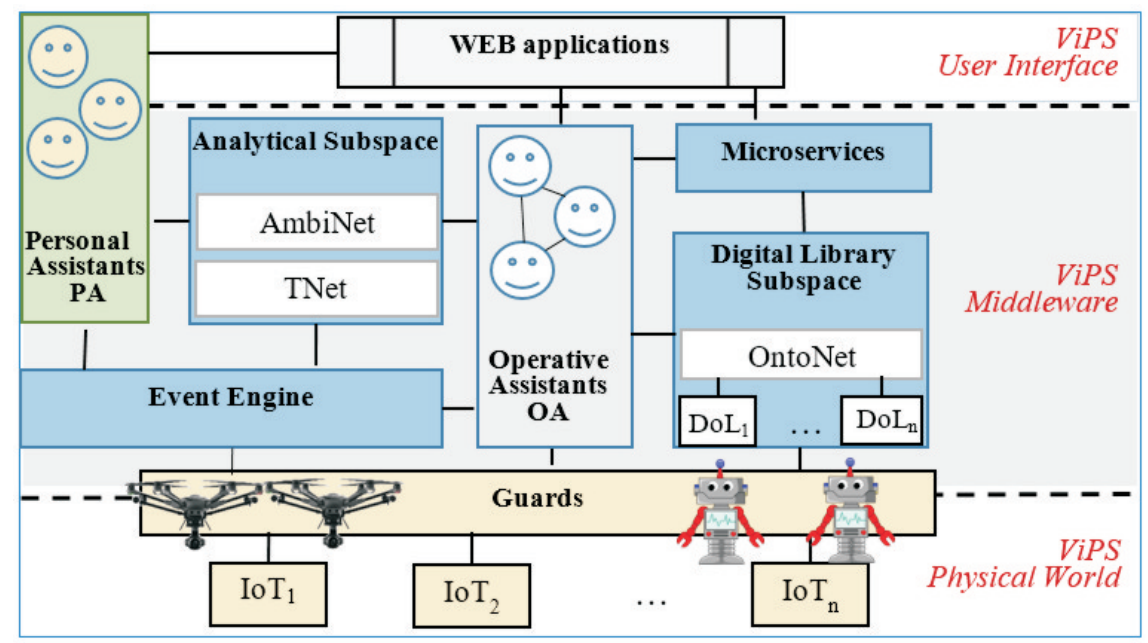

Fig. 1. Architecture of ViPS. (c) 2018 IEEE

The personal assistants must also be able to operate in an "overtaking action" mode, i.e. as an early warning system. In this way, certain operations must be performed before the event occurs. Therefore, the personal assistants cannot rely on the Event Engine in the timeframe before the event occurs. Then, they are supported by the AmbiNet and Temporal Net (TNet) components. The interaction between the personal assistants and the Analytical Subspace is implemented by specialized interaction protocols. Additionally, the user interface can be supplemented with a web application. The web application could offer public information resources of the space as well. Several components extremely essential for the operation of ViPS are located in the middle layer of the architecture. Practically, the virtualization of "things" is supported by this level. The middleware takes into account time and spatial aspects of the "things" to be virtualized as well the events binding "things" with user's activities. In this way, the Analytical Subspace provides means for the preparation of a domain-specific analysis based on the following formalisms and the supported tools:

- Interval Temporal Logics [31] and TNet - provide an possibility to present and work with the temporal aspects of things, events and locations;

- Calculus of Context-aware Ambients [32] and AmbiNet - the spatial characteristics of the "things" and the events are modelled as ambients;

- Event Model [33] and Event Engine - model and interpret various types of events and their arguments such as identification, conditions for occurrence and completion. 
The components of the Analytical Subspace are supported by the Digital Libraries Subspace operating as a central repository of ViPS. This subspace stores domain-specific background knowledge and documentation. The repository is implemented as a knowledge network including relational data bases and a hierarchy of ontologies known as OntoNet.

The operative assistants implemented as rational intelligent agents provide access to the resources of the two subspaces and they accomplish interactions with the personal assistants and the web applications. They are architectural components suitable for providing the necessary dynamism, flexibility, and intelligence. Unfortunately, the agents are unsuitable to implement business functionality. For this reason, assistants work closely with services or microservices.

The Guards operate as an intelligent interface between the virtual and the physical environments. They provide data about the state of the physical environment transferred to the virtual part of ViPS. There are multiple IoT nodes integrated in the architecture of the guards that provide access to sensors and actuators of the "things" located in the physical environment. The sensors-actuators configurations are domain-specific. The communication in the guard system operates as a combination of a personal network (e.g. LoRa) and the Internet.

Usually, a ViPS-like application for a specific domain needs only some ViPS components to be adapted. Subsequently, these adapted components are archived in the Domain Libraries. In this way, the genetic architecture can be continuously ended. The smart agriculture aims to help address three major challenges: an efficient use of water for irrigation, an effective pest control and, at the same time, minimizing the risk of environmental pollution by pesticides, and an early detection of plant diseases. The space consists of the following four components: Operative Center, DiLib "Smart Agriculture", Operative Assistants, and Guard Network, Fig. 2.

ViPS adapted for smart agriculture consists of the following basic components: Analytical Subspace (including Personal Assistants), Operative Subspace, Agriculture Data and Knowledge Center, Guards Subspace, and External Interfaces, Fig. 3.

The Agriculture Data and Knowledge Center (ADK Center) stores background knowledge about agriculture and data obtained from the sensor networks located in the physical world. In this center, the objects are presented as instances of two structural templates. The first one known as static (or features) template contains the inherent attributes (characteristics) of the objects of interest. The second one called dynamic template (or meta-template) 


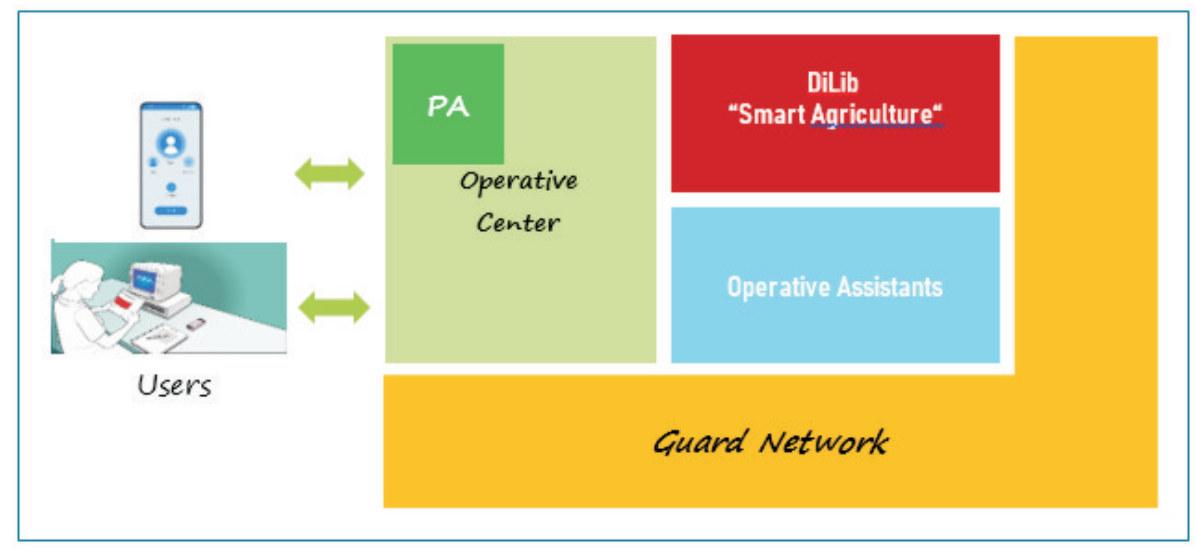

Fig. 2. Smart Agriculture Space

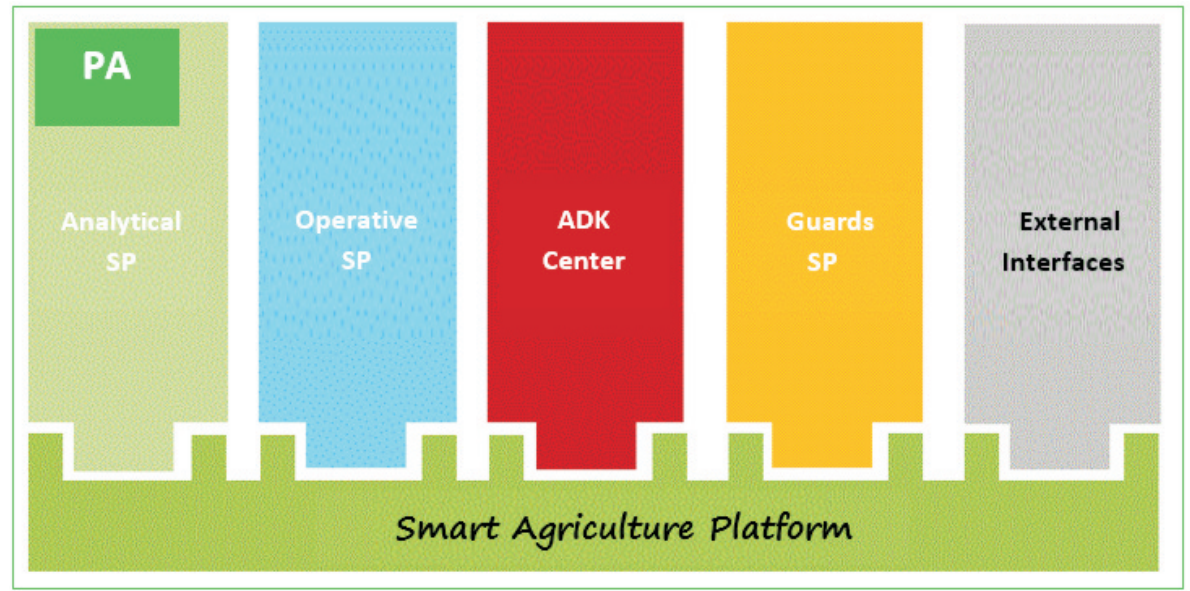

Fig. 3. Smart Agriculture Platform

stores information about spatial, temporal and event aspects related to the respective object. Basically, the information is stored in ontologies and relational databases mutually integrated. The ADK Center provides also tools for receiving, storing, and processing large volume of structured, semi-structured, and unstructured agriculture data.

The Analytical Subspace is the operative center assisting agricultural operators in managing, controlling and coordinating all stages of needed activities. For this purpose, virtualization is mainly performed here, supported by various modelling environments located in this subspace. Currently, the temporal aspects of the things are modelled through the TNet component implemented 
on the basis of the Interval Temporal Logics [31]. The events in the analytical subspace are specified in accordance with the Event Model and are interpreted by the corresponding Event Engine [33].

Cyber-Physical and Social Systems processes due to complexity require prior modelling and verification. It is necessary to consider the spatial aspects of intelligent IoTs when developing a system for intelligent agriculture. For this purpose, ambient-oriented Calculus of Context-aware Ambients (CCA) modelling is used for the implementation of which a special tool is created - a visual CCA editor, through which the basic scenarios in the smart agriculture system are modelled and verified. A system for intelligent agriculture is built on the basis of the reference ViPS architecture [35]. Preliminary modelling, testing, and verification of processes and scenarios are part of the main functionalities of the Analytical Subspace. The first prototype version of the visual editor developed for the CCA modelling significantly accelerates this process.

Some problems regarding the development and use of the event model are solved in [36]. The first issue concerns the implementation of Event Engine 2.0 that is based on its predecessor. The idea is to keep the existing representation model while improving the event distribution mechanism, making it more natural for use by the rational agents and thus reducing their development time and complexity. The consequence is a library that contains a set of behaviours, configuration utilities, communication protocols, the ontology and the default realization of the rational agent with event brokering functionality. Event Engine 2.0 supports a proactive management of events; instead of demanding every agent to check for new events, the engine reverses the process by compelling the new events to inform the interested parties about their existence. When a new event occurs, it is represented by its own agent that declares its existence to the other agents in the system. As the number of events is growing, this approach is becoming relatively inefficient even in environments that are highly optimized to support a lot of agent instances. Alternatively, a single agent is designed to represent a whole category of events. Furthermore, the event model permits the event categories to inherit each other, which allows filtering and reduction of the communication traffic only to those categories that a particular party is interested in. The more general the category, the greater the number of events.

Architecture for a knowledge base in the field of intelligent plant agriculture is designed in [37] to automate the work on growing different types of agricultural crops. One of the main tasks is to make the process of cultivation more predictable, which will help farmers obtain better quality crops. For the realization of the architecture, the authors have chosen Protégé for the 
ontologies, JaCaMo for the environment of the multi-agent system, Jason for the personal assistant, and Jade for the operational assistants.

In [38], it is shown that the regional data center is an important component related to the development of intelligent agriculture systems. It maintains the main part of the data related to the cultivation of crops in the region. Based on the collected data, analyses and predictions can be made easily for the production and development of agricultural activities for the region of Plovdiv. It provides basic data on the operation of the ZEMELA platform, which includes intelligent components to support farmers in making decisions about growing crops and implementing preventive measures to avoid various problems. The analyses and statistics obtained on the basis of the data collected in the regional data center for intelligent agriculture can be useful for decisionmaking at the national level as well. This research will contribute to the more sustainable management of natural resources, to reduce the harmful effects of agriculture on the environment and the climate, to decrease the use of pesticides and increase the quality and safety of agricultural products, thus ensuring food security and public health.

An environment for modelling scenarios and processes in intelligent agriculture settings, known as ViSMod, is presented in [39]. ViSMod is developed as an expert system, the architecture of which is also given in this paper. The use of the environment is demonstrated by an example - a system for diagnosing possible poisoning of ruminants. The diagnostic system presented in the article is implemented on SWI-Prolog.

The paper [40] presents a platform called ZEMELA. ZEMELA is developed as a cyber-physical-social space providing various services for intelligent agriculture. The general architecture of the platform is briefly described. Furthermore, the kernel of the platform consisting of an event model and personal assistants is also presented. One example demonstrates the interaction between the two components of the platform. Currently, the first version of the platform is under development. The platform ZEMELA is implemented by the help of agent-oriented development environment JaCaMo.

In [41], the developed supply chain model connects the main participants in the experimental network and provides the possibility of implementing the described interactions between them through the use of blockchain technology and the creation of different channels of interaction of different types.

Blockchain models of the individual channels have been developed and their prototype implementations are being worked on. Currently, a three-layer infrastructure model of the intelligent environment for intelligent agriculture is being built, which includes processing of dynamically incoming IoT data at 
the sensory level and their processing in regional and national cloud data centers. This will provide an possibility of implementing the prototype, testing, and verification of the modelled supply chain.

A conceptual framework for group multi-criteria selection of blockchain software in fuzzy environment according to organization needs and experts' judgements is proposed in [42]. The applicability of the new framework has been verified through an illustrative example for ranking blockchain systems. The evaluations of compared alternatives were calculated by using measurement of alternatives and ranking according to the compromise solution (MARCOS) method. The robustness of the new framework was proven by sensitivity analysis in which two (crisp and fuzzy) MARCOS models with two different sets of weighting coefficients were compared.

In [43], it is proposed a reverse interpretation of the worst-case solutions as recommendation for preliminary guideline for risk management recourse allocation between these elements of the Enterprise Global Risk Management (EGRM). The presented results support the assumption that EGRM framework is applicable as an additional interpretive tool for risk management decision-making in blockchain deployment. The framework could be considered as promising tool for ex-ante guidelines for allocating resources (financial or other) of enterprises/organizations among the elements of the EGRM.

The paper [44] presents the main issues regarding competencies and knowledge, risks and challenges in performing internal audit when adopting blockchains. The main purpose is to propose a framework for stages, procedures and elements for an internal audit plan in organizations that implement blockchains in agriculture. The basic elements of blockchain technology, characteristics and functions of internal audit and internal control are analyzed, as well as the impact of blockchains implementation on organization and focus areas affecting human, financial and technical resources, risk identification, blockchain control procedures, risk management and mitigation. An example of suggested framework for internal audit plan is presented.

\section{CONCLUSIONS}

Plant genetic resources for food and agriculture are critically important for the sustainable food production in conditions of an ever-growing population and climate change. Intelligent agriculture aims to improve the quantity and quality of yields by tackling three main challenges: efficient use of water for irrigation, effective pest control and at the same time minimizing the risk of pesticide pollution, and early detection of plant diseases. To achieve this goal 
with the help of information and communication technologies it is necessary to look for appropriate solutions and technological platforms.

This paper presents the results of research aimed at the application of artificial intelligence to support Bulgarian crop production. The results obtained within the framework of the project BG PLANTNET "Establishment of National Information Network GenBank - Plant genetic resources" and the National Research Program "Smart crop production" are presented. The reference architecture is presented, as well its adaptation for an important domain, namely smart crop production. The virtualization of the physical objects of interest, which could be a crucial way for the integration of the virtual and physical worlds, is indicated as a serious challenge.

Future research will focus on expanding the scope of artificial intelligence in various areas of agricultural production. The Smart Agriculture Space will be supported by combination of created various approaches to modelling and developed technological platforms.

\section{ACKNOWLEDGEMENTS}

This work is supported by the Bulgarian Ministry of Education and Science under the National Research Program "Smart crop production", Grant Д01-65/19.03.2021, approved by Decision of the Ministry Council No 866/ 26.11.2020 and partly supported by the Bulgarian National Science Fund of the Ministry of Education under the project BG PLANTNET "Establishment of National Information Network GenBank - Plant genetic resources", Grant КП-06H36/2.

\section{REFERENCES}

[1] National Scientific Program "Intelligent Crop Production", www.nnp-ir.bg.

[2] T. Glushkova, S. Stoyanov, L. Doukovska, J. Todorov, and I. Stoyanov, Modelling of an Irrigation System in a Virtual Physical Space, Mathematical Biosciences and Engineering, Special Issue on Intelligent DataCentric Systems, AIMS Press (2021) 18 (5) 6841-6856, ISSN: 1551-0018, DOI: 10.3934/mbe.2021340.

[3] E. Doychev, P. Malinov, N. Velcheva, and Z. Duchev, A Genbank Architecture, A Distributed System for Management of Plant Genetic Resources, in: Proceedings of the $10^{\text {th }}$ International Conference on Intelligent Systems IS'20, Varna, Bulgaria, IEEE Xplore, 2020, pp. 580-583, ISBN: 978-1-7281-54565, ISSN: 1541-1672, DOI: 10.1109/IS48319.2020.9199972. 
[4] T. Glushkova, S. Stoyanov, I. Popchev, and L. Doukovska, AmbientOriented Modelling in an Intelligent Agriculture Infrastructure, in: Proceedings of the $10^{\text {th }}$ International Conference on Intelligent Systems - IS'20, Varna, Bulgaria, IEEE Xplore, 2020, pp. 612-618, ISBN: 978-1-7281-5456-5, ISSN: 15411672, DOI: 10.1109/IS48319.2020.9199952.

[5] A. Stoyanova-Doycheva, V. Ivanova, E. Doychev, and K. Spassova, Development of an Ontology in Plant Genetic Resources, in: Proceedings of the $10^{\text {th }}$ International Conference on Intelligent Systems - IS'20, Varna, Bulgaria, IEEE Xplore, 2020, pp. 246-251, ISBN: 978-1-7281-5456-5, ISSN: 1541-1672, DOI: $10.1109 /$ IS48319.2020.9199935.

[6] I. Stoyanov, A. Stoyanova-Doycheva, I. Krasteva, and Z. Uhr, A Personal Assistant Supporting Agriculture Operators, in: Proceedings of the $10^{\text {th }}$ International Conference on Intelligent Systems - IS'20, Varna, Bulgaria, IEEE Xplore, 2020, pp. 584-589, ISBN: 978-1-7281-5456-5, ISSN: 1541-1672, DOI: 10.1109/IS48319.2020.9199953.

[7] I. Krasteva, T. Glushkova, N. Moraliyska, and N. Velcheva, A Blockchain Based Model of Genbank Store System, in: Proceedings of the $10^{\text {th }}$ International Conference on Intelligent Systems - IS'20, Varna, Bulgaria, IEEE Xplore, 2020, pp. 606-611, ISBN: 978-1-7281-5456-5, ISSN: 1541-1672, DOI: 10.1109/IS48319.2020.9200133.

[8] I. Popchev and D. Orozova, Text Mining in the Domain of Plant Genetic Resources, in: Proceedings of the $10^{\text {th }}$ International Conference on Intelligent Systems - IS'20, Varna, Bulgaria, IEEE Xplore, 2020, pp. 596-600, ISBN: 9781-7281-5456-5, ISSN: 1541-1672, DOI: 10.1109/IS48319.2020. 9200174.

[9] I. Popchev and I. Radeva, Decision Making Model for Disruptive Technologies in Agriculture, in: Proceedings of the $10^{\text {th }}$ International Conference on Intelligent Systems - IS'20, Varna, Bulgaria, IEEE Xplore, 2020, pp. 258-264, ISBN: 978-1-7281-5456-5, ISSN: 1541-1672, DOI: 10.1109/IS48319.2020.9199962.

[10] G. Illeva, Cloud Technologies: Multi-criteria Selection, Academic Publishing House "Prof. Marin Drinov" (2020) 136, ISBN: 978-619-245-079-3.

[11] I. Popchev And D. Orozova, Data Analytics Approach in Virtual Educational Space, in: Proceedings of the IEEE International Conference on Big Data, Knowledge and Control Systems Engineering - BdKCSE'2021, Sofia, Bulgaria, IEEE Xplore, October 28-29, 2021 (in print).

[12] V. Sgurev, L. Doukovska, and St. Drangajov, Making Optimal Intelligent Solutions with Risks in Transportations Networks for Haulage of Agricultural Products, in: Proceedings of the $10^{\text {th }}$ International Conference on Intelligent Systems - IS'20, Varna, Bulgaria, IEEE Xplore, 2020, pp. 270-275, ISBN: 9781-7281-5456-5, ISSN:1541-1672, DOI: 10.1109/IS48319.2020.9199984.

[13] A. Toskova, B. Toskov, Z. Uhr, And L. Doukovska, Recognition of Wheat Pests, in: Proceedings of the $10^{\text {th }}$ International Conference on Intelligent Systems - IS'20, Varna, Bulgaria, IEEE Xplore, 2020, pp. 276-280, ISBN: 978-1-72815456-5, ISSN: 1541-1672, DOI: 10.1109/IS48319.2020.9200148. 
[14] V. Valkanov, M. Petrov, D. Rusev, and I. Radeva, Modelling Distributed Fault-Tolerant High Availability Storage Cluster Based on Block-Chain Concepts for Tracking Scientific-Research Progress, in: Proceedings of the $10^{\text {th }}$ International Conference on Intelligent Systems - IS'20, Varna, Bulgaria, IEEE Xplore, 2020, pp. 590-595, ISBN: 978-1-7281-5456-5, ISSN: 1541-1672, DOI: 10.1109/IS48319.2020.9199980.

[15] A. Stoyanova-Doycheva, E. Doychev, S. Stoyanov, And A. Toskova, An Intelligent GeneBank Management System, in: Proceedings of the International Conference Automatics and Informatics - ICAI'20, Varna, Bulgaria, IEEE Explore, October 1-3, 2020, DOI: 10.1109/ICAI50593.2020.9311295.

[16] N. Velcheva, K. Uzundzhalieva, and S. Cheperigova, Documentation of Plant Genetic Resources in Bulgaria - Current Status and New Approaches, in: $3^{\text {rd }}$ International Conference on Food, Agriculture and Veterinary, Ege University, Izmir, Turkey, June 19-20, 2021, pp. 415-421, ISBN: 978-625-7720-43-4.

[17] W. Wolf, Cyber-Physical Systems, Computer (2009) 42 (3) 88-89.

[18] Kim Kyoung-Dae And P. R. Kumar, Cyber-Physical Systems: A Perspective at the Centennial, in: Proceedings of the IEEE (Special Centennial Issue), 2012, 100, 1287-1308.

[19] R. R. Rajkumar, I. Lee, L. Sha, and J. Stankovic, Cyber-Physical Systems: The Next Computing Revolution, in: Proceedings of the $47^{\text {th }}$ Design Automation Conference, ACM, 2010, pp. 731-736.

[20] L. Atzori, A. Iera, And G. Morabito, The Internet of Things: A Survey, Computer Networks (2010) 54 2787-2805.

[21] J. Zheng, D. Simplot-Ryl, C. Bisdikian, and H. T. Mouftah, The Internet of Things, IEEE Communications Magazine (November 2011) 49 30-31.

[22] D. Miorandi, S. Sicari, Francesco De Pellegrini, and Imrich ChlamTAC, Internet of Things: Vision, Applications and Research Challenges, Ad Hoc Networks (2012) 10 (7) 1497-1516.

[23] Jianhua Shi, Jiafu Wan, Hehua Yan, and Hui Suo, A Survey of CyberPhysical Systems, in: IEEE WCSP'11, 2011, pp. 1-6.

[24] F. Y. WANG, The Emergence of Intelligent Enterprises: From CPS to CPSS, IEEE Intelligent Systems (July/Aug. 2010) 25 (4) 85-88.

[25] K. Kirkpatrick, Technologizing Agriculture, Communications of the ACM (2019) 62 (2) 14-16.

[26] M. Kang, X. R. Fan, J. Hua, H. Wang, X. Wang, and F. Y. Wang, Managing Traditional Solar Greenhouse with CPSS: A Just-for-Fit Philosophy, IEEE Transaction on Cybernetics (2018) 12 3371-3380.

[27] F. Kiani And A. Seyyedabbasi, Wireless Sensor Network and Internet of Things in Precision Agriculture, International Journal of Advanced Computer Science and Applications (2018) 9 (6) 99-103. 
[28] C. D. Moreno-Moreno M. Brox-Jiménez, A. A. Gersnoviez-Milla, M. Márquez-Moyano, M. A. Ortiz-López, and F. J. Quiles-Latorre, Wireless Sensor Network for Sustainable Agriculture, in: Proceedings of the Green Technology and Engineering International Conference GTEIC'18, Caceres, Spain, 2018, 2, 1302, DOI:10.3390/proceedings2201302.

[29] F. Karim And A. Frinida, Monitoring System Using Web of Things in Precision Agriculture, Elsevier, Procedia Computer Science (2017) $110402-409$.

[30] A. Kamilaris, A. Kartakoullis, and F. X. Prenafeta-Boldú, A Review on the Practice of Big Data Analysis in Agriculture, Computers and Electronics in Agriculture (2017) 143 23-37.

[31] B. Moszkowski, Compositional Reasoning Using Interval Temporal Logic and Tempura, Springer, Lectures Notes in Computer Sciences (1998) 1536 439-464.

[32] F. Siewe, H. Zedan, And A. Cau, The Calculus of Context-Aware Ambients, Journal of Computer and System Sciences (2010) 77 (4) 597-620.

[33] Z. Guglev and S. Stoyanov, Hybrid Approach for Manipulation of Events in the Virtual Referent Space, in: Proceedings of the International Scientific Conference Blue Economy and Blue Development, Burgas Free University, Burgas, Bulgaria, 2018, pp. 197-203, ISBN 978-619-7126-57-0.

[34] M. Wooldridge, An Introduction to Multi Agent Systems, Wiley, 2009, ISBN 978-0-470-51946-2.

[35] T. Glushrova, S. Stoyanov, K. Rusev, I. Krasteva, and N. MoraliYska, Ambient-Oriented CCA Modelling in Agriculture, in: Proceedings of the IEEE International Conference on Automatics and Informatics - ICAI'21, Varna, Bulgaria, IEEE Xplore, 30 September-2 October, 2021 (in print).

[36] S. Stoyanov, A. Stoyanova-Doycheva, V. Ivanova, V. TabakovaKomsalova, V. Monov, and Z. Radeva, An Event Model for Smart Agriculture, in: Proceedings of the IEEE International Conference on Automatics and Informatics - ICAI'21, Varna, Bulgaria, IEEE Explore, 30 September-2 October, 2021 (in print).

[37] A. Stoyanova-Doycheva, V. Ivanova, L. Doukovska, V. Tabakova, I. Radeva, and S. Danailova, Architecture of a Knowledge Base in Smart Crop Production, in: Proceedings of the IEEE International Conference on Automatics and Informatics - ICAI'21, Varna, Bulgaria, IEEE Xplore, 30 September-2 October, 2021 (in print).

[38] E. Doychev, A. Terziyski, P. Atanasova, O. Rahneva, V. Ivanova, and A. Stoyanova-Doycheva, A Regional Data Center for Intelligent Agriculture, in: Proceedings of the IEEE International Conference on Big Data, Knowledge and Control Systems Engineering - BdKCSE'2021, Sofia, Bulgaria, IEEE Xplore, October 28-29, 2021 (in print). 
[39] V. Tabakova-Komsalova, L. Doukovska, I. Stoyanov, J. Todorov, S. Stoyanov, and Z. Radeva, ViSMod - An Environment for Modelling of Scenarios and Processes in Intelligent Agriculture, in: Proceedings of the IEEE International Conference on Big Data, Knowledge and Control Systems Engineering - BdKCSE'2021, Sofia, Bulgaria, IEEE Xplore, October 28-29, 2021 (in print).

[40] S. Stoyanov, J. Todorov, I. Stoyanov, V. Tabakova-Komsalova, L. Doukovska, AND A. Dukovski, ZEMELA - An Intelligent Agriculture Platform, in: Proceedings of the IEEE International Conference on Big Data, Knowledge and Control Systems Engineering - BdKCSE'2021, Sofia, Bulgaria, IEEE Xplore, October 28-29, 2021 (in print).

[41] I. Krasteva, T. Glushkova, A. Stoyanova-Doycheva, N. Moraliyska, L. Doukovska, And I. Radeva, Blockchain Based Approach to Supply Chain Modelling in a Smart Farming System, in: Proceedings of the IEEE International Conference on Big Data, Knowledge and Control Systems Engineering BdKCSE'2021, Sofia, Bulgaria, IEEE Xplore, October 28-29, 2021 (in print).

[42] G. Ilieva, T. Yankova, I. Radeva, and I. Popchev, Blockchain Software Selection as a Fuzzy Multi-Criteria Problem, Computers (2021) 10 (10), DOI:10.3390/computers10100120.

[43] I. Popchev, I. Radeva, and V. Velichkova, Blockchains in Enterprise Global Risk Management, in: Proceedings of the IEEE International Conference on Automatics and Informatics - ICAI'21, Varna, Bulgaria, IEEE Xplore, 30 September-2 October, 2021 (in print).

[44] I. Popchev, I. Radeva, ANd V. VelichKova, The Impact of Blockchain on Internal Audit, in: Proceedings of the IEEE International Conference on Big Data, Knowledge and Control Systems Engineering - BdKCSE'2021, Sofia, Bulgaria, IEEE Xplore, October 28-29, 2021 (in print). 